\title{
Finite-SNR Diversity-Multiplexing Tradeoff for Two-Way Relay Fading Channel
}

\author{
Xiaochen $\mathrm{Lin}^{\dagger}$, Meixia $\mathrm{Tao}^{\dagger}$, Youyun $\mathrm{Xu}^{\dagger}$ and Xiaodong Wang* \\ ${ }^{\dagger}$ Dept. of Electronic Engineering, Shanghai Jiao Tong University, Shanghai 200240, China \\ * Dept. of Electrical Engineering, Columbia University, New York, NY 10027, USA \\ Emails: \{xclin_comm,mxtao\}@sjtu.edu.cn, yyxu@vip.sina.com, wangx@ee.columbia.edu
}

\begin{abstract}
This paper studies the performance limits of twoway relay channel (TWRC) at finite signal-to-noise ratio (SNR) in Rayleigh fading environment. A two-phase decode-and-forward (DF) protocol is considered. We first derive closed-form expressions for both outage probability and diversity-multiplexing tradeoff (DMT). Our results are general and suitable for any time sharing and any rate allocation in the two-way relay protocol. It is found that DF outperforms amplify-and-forward (AF) when either multiplexing gain or SNR is small enough, otherwise, DF is inferior to AF in term of outage probability. Meanwhile, finiteSNR DMT of DF is always lower than that of AF regardless of SNR due to the additional sum-rate constraint imposed on the relay node for full decoding. Furthermore, the optimum relay location for any given combination of time sharing and rate allocation is presented.
\end{abstract}

\section{INTRODUCTION}

Thanks to its great potential in enhancing spectral efficiency, two-way relay channel (TWRC), where two source nodes exchange information with the help of a relay node, has attracted a lot of interests [1]-[7]. Several relay protocols have been proposed, including two-phase amplify-and-forward (AF) [4], two-phase and three-phase decode-and-forward (DF) [5] [6] [7]. A common performance metrics applied in these works to evaluate these protocols is achievable rate region, which however is for deterministic channels. For wireless fading channels, new performance metrics are desired for further understanding of the performance limits of two-way relaying.

Diversity-multiplexing tradeoff (DMT), firstly proposed by Zheng and Tse [8] has become a fundamental and essential tool to analyze the performance of not only multiple-input multiple-output (MIMO) fading channels but also relay fading channels. Recently, the authors in [9] and [10] present the DMT analysis for both half-duplex and full-duplex TWRC with DF and compress-and-forward protocols. It is noted that these studies are for asymptotically high SNR region. However, practical communication systems, such as wireless local area network and cellular networks usually operate in the low and medium SNR range. More importantly, it is only in the low and medium SNR region that relay-assisted transmission will play an important role (if SNR is high enough, direct transmission is preferred). Hence, it is more desirable to

This work is supported by the National Natural Science Foundation of China under grant 60902019, by the National High-Tech R\&D Program (863 Program) Key Project of China under grant 2009AA011505, and by the Natural Science Foundation of Shanghai under grant 08ZR1410400.

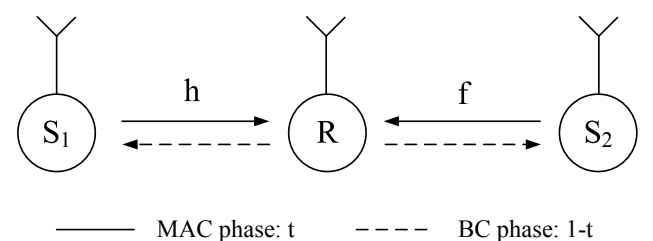

Fig. 1. System model of TWRC

analyze DMT at finite SNR range as presented in [11]. Given that, for quasi-static fading channels, the packet error rate is equal to the outage probability with the capacity-achieving codes applied, the authors in [11] present the new definitions of diversity gain and multiplexing gain at finite SNR. The multiplexing gain determines how the target date rate scales with a finite SNR, while diversity gain at a particular SNR is used to predict the additional SNR required to decrease a specified amount of outage probability for a given multiplexing gain. Recently, authors in [12] extend the finite-SNR DMT analysis for TWRC. But it is for AF protocol only.

In this paper, we complement the literature of DMT analysis for TWRC at finite SNR by considering two-phase DF protocol. Closed-form expressions for both outage probability and finite-SNR DMT in Rayleigh fading channels are derived. Note that the priori work in [12] only considers equal time sharing and fair rate allocation. Our results are general and suitable for any time sharing and rate allocation in the twoway relay protocol. Through numerical study, the optimum relay location under certain conditions on time sharing and rate allocation is discussed as well.

\section{SySTEM MOdEL}

Consider a TWRC shown in Fig. 1, where two sources $S_{1}$ and $S_{2}$ communicate with each other aided by the relay $R$. It is supposed that every terminal is equipped with single antenna and has the same transmission power $E$ (the extension to different power is straightforward). Each link is assumed to be reciprocal, i.e., the channel coefficients between $\mathrm{S}_{1}$ and $\mathrm{R}$, and between $\mathrm{S}_{2}$ and R satisfy $h_{1 R}=h_{R 1}=h, f_{2 R}=f_{R 2}=f$. Here both $h$ and $f$ are zero-mean complex Gaussian random variables with variances $\Omega_{h}$ and $\Omega_{f}$, respectively.

The bidirectional transmission takes place in two phases using DF relay protocol. In the first phase, also called multipleaccess (MA) phase, $S_{1}$ and $S_{2}$ transmit simultaneously towards $\mathrm{R}$ for a time fraction of $t$ and the relay attempts to jointly 
decode the two signals. Assume the noise in all links is additive Gaussian with zero mean and unit variance. Then, the instantaneous SNRs of the links between the two source nodes and the relay node can be denoted as $\gamma_{1 R}=E|h|^{2}$ and $\gamma_{2 R}=E|f|^{2}$, respectively. The instantaneous total received SNR at $\mathrm{R}$ is $\gamma_{\mathrm{mac}}=E|h|^{2}+E|f|^{2}$. To successfully decode both signals from two sources at the relay, the achievable rate pair $\left(R_{1}, R_{2}\right)$ of the two sources needs to satisfy the following MA channel constraints:

$$
\begin{aligned}
R_{1} & \leq I_{1 R} \triangleq t \log _{2}\left(1+\gamma_{1 R}\right), \\
R_{2} & \leq I_{2 R} \triangleq t \log _{2}\left(1+\gamma_{2 R}\right), \\
R_{1}+R_{2} & \leq I_{\mathrm{mac}} \triangleq t \log _{2}\left(1+\gamma_{\mathrm{mac}}\right)
\end{aligned}
$$

In the second phase, named as broadcast (BC) phase, the relay $\mathrm{R}$, after applying ideal random binning, transmits the combined signals towards the two desired destinations for a time fraction of $1-t$. Note that the total transmission time of the considered two-phase protocol is normalized to one and we refer to $t$ as the time-sharing parameter. In the BC phase, to ensure successful decoding at both terminals, the achievable rate pair $\left(R_{1}, R_{2}\right)$ needs to satisfy [6]:

$$
\begin{aligned}
& R_{1} \leq I_{R 2} \triangleq(1-t) \log _{2}\left(1+\gamma_{2 R}\right) \\
& R_{2} \leq I_{R 1} \triangleq(1-t) \log _{2}\left(1+\gamma_{1 R}\right)
\end{aligned}
$$

Applying the cut-set bound theory, the achievable rate region of TWRC is then given by all the possible rate pairs $\left(R_{1}, R_{2}\right)$ satisfying:

$$
\begin{aligned}
R_{1} & \leq \min \left(I_{1 R}, I_{R 2}\right), \\
R_{2} & \leq \min \left(I_{2 R}, I_{R 1}\right), \\
R_{1}+R_{2} & \leq I_{\mathrm{mac} .}
\end{aligned}
$$

\section{OUtAGE PROBABILITY}

To obtain finite-SNR DMT, we derive the outage probability first in this section. Let $R$ denote the target sum-rate of TWRC. The target rates of source $\mathrm{S}_{1}$ and $\mathrm{S}_{2}$ are $\alpha R$ and $(1-\alpha) R$, respectively, where $\alpha$ is called the rate allocation parameter. The TWRC system is said to be in outage when any source transmission is in outage. In response to the rate constraints (6)-(8), we define three events:

$$
\left\{\begin{array}{l}
A_{1}: \alpha R \leq \min \left(I_{1 R}, I_{R 2}\right) \\
A_{2}:(1-\alpha) R \leq \min \left(I_{2 R}, I_{R 1}\right) . \\
A_{3}: R \leq I_{\mathrm{mac}}
\end{array}\right.
$$

Then, the outage probability of the TWRC system can be expressed as:

$$
P_{\text {out }}=1-P\left(A_{1} \cap A_{2} \cap A_{3}\right) .
$$

Substituting the definitions of $I_{i R}, I_{R i}$ and $I_{\mathrm{mac}}$, for $i=1,2$ in (1)-(5) into the events (9) and after simple manipulation, we obtain the equivalent conditions on the channel gain pair

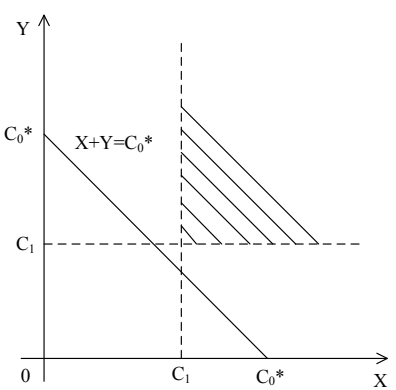

(a) $2 C_{1}>C_{0}^{*}$

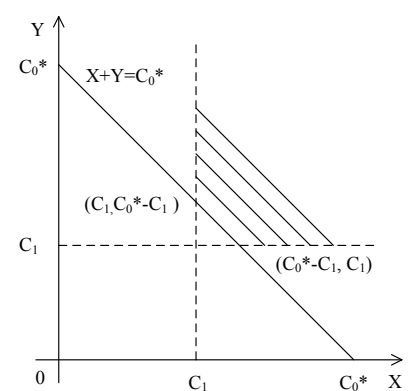

(b) $2 C_{1} \leq C_{0}^{*}$
Fig. 2. Channel gain pair $(X, Y)$

$\left(X \triangleq|h|^{2}, Y \triangleq|f|^{2}\right)$ for reliable communications as

$$
\left\{\begin{array}{l}
X \geq \max \left(\frac{2^{\frac{\alpha R}{t}}-1}{E} \triangleq C_{X 1}, \frac{2^{\frac{(1-\alpha) R}{1-t}}-1}{E} \triangleq C_{X 2}\right) \\
Y \geq \max \left(\frac{2^{\frac{\alpha R}{1-t}}-1}{E} \triangleq C_{Y 1}, \frac{2^{\frac{(1-\alpha) R}{t}}-1}{E} \triangleq C_{Y 2}\right) \\
X+Y \geq \frac{2^{\frac{R}{t}}-1}{E} \triangleq C_{0}
\end{array} .\right.
$$

Here, both channel gains $X$ and $Y$ follow exponential distribution with mean $\Omega_{h}$ and $\Omega_{f}$, respectively. In (11), both time-sharing parameter $t$ and rate allocation parameter $\alpha$ are considered. In the following three subsections, we derive the probability of the joint event (11) and obtain the outage probability when both parameters take different values.

\section{A. Equal time sharing and arbitrary rate allocation}

For simplicity, we first assume equal time sharing, i.e., $t=$ $1 / 2$. Thus, (11) becomes $\left\{(X, Y) \mid X \geq \max \left(\frac{2^{2 \alpha R}-1}{E} \triangleq\right.\right.$ $\left.C_{1}, \frac{2^{2(1-\alpha) R}-1}{E} \triangleq C_{2}\right), Y \geq \max \left(C_{1}, C_{2}\right), X+Y \geq \frac{2^{2 R}-1}{E} \triangleq$ $\left.C_{0}^{*}\right\}$. Without loss of generality, we let the target rate of $S_{1}$ is greater than or equal to the target rate of $\mathrm{S}_{2}$, i.e., $1 / 2 \leq \alpha \leq 1$. Thus $C_{1} \geq C_{2}$. Hence, after further simplification, $(X, Y)$ satisfies $\left\{(X, Y) \mid X \geq C_{1}, Y \geq C_{1}, X+Y \geq C_{0}^{*}\right\}$.

Depending on the value of $C_{0}^{*}$ in comparison with $C_{1}$, the region of the channel gain pair $(X, Y)$ that satisfies the above conditions can be plotted in Fig. 2, as shown by shadowed area. Thus, using the probability distribution function of exponential variables and considering that $X$ and $Y$ are independent, we can obtain the outage probability of the TWRC system as:

$$
= \begin{cases}P_{\text {out }(\alpha, \mathrm{t}=1 / 2)} & \alpha_{0} \leq \alpha \leq 1 \\ 1-\frac{\Omega_{h}}{\Omega_{h}-\Omega_{f}} e^{-\frac{1}{\Omega_{h}} C_{0}^{*}+\left(-\frac{1}{\Omega_{f}}+\frac{1}{\Omega_{h}}\right) C_{1}} & \\ +\frac{\Omega_{f}}{\Omega_{h}-\Omega_{f}} e^{-\frac{1}{\Omega_{f}} C_{0}^{*}+\left(-\frac{1}{\Omega_{h}}+\frac{1}{\Omega_{f}}\right) C_{1}}, & \frac{1}{2} \leq \alpha \leq \alpha_{0}\end{cases}
$$

where $\alpha_{0} \triangleq\left(\log _{2}\left(2^{2 R}+1\right)-1\right) / 2 R$ is the root of $2 C_{1}=C_{0}^{*}$, i.e., $2 \cdot\left(2^{2 \alpha R}-1\right)=2^{2 R}-1$, and is shown to be greater than or equal to $1 / 2$. In the special case where the two channel statistics are the same, i.e., $\Omega_{h}=\Omega_{f}$, the outage probability 
is:

$$
P_{\text {out }(\alpha, \mathrm{t}=1 / 2)}= \begin{cases}1-e^{-\frac{2}{\Omega_{h}} C_{1}}, & \alpha_{0} \leq \alpha \leq 1 \\ 1-e^{-\frac{1}{\Omega_{h}} C_{0}^{*}} & \\ -\frac{1}{\Omega_{h}}\left(C_{0}^{*}-2 C_{1}\right) e^{-\frac{1}{\Omega_{h}} C_{0}^{*}}, & \frac{1}{2} \leq \alpha \leq \alpha_{0}\end{cases}
$$

\section{B. Arbitrary time sharing and equal rate allocation}

Next, let the rate allocation be fair, i.e., the target rate of each source is $R / 2$. Then, (11) is simplified as $\{(X, Y) \mid X \geq$ $\max \left(\frac{2^{\frac{R}{2 t}}-1}{E} \triangleq C_{3}, \frac{2^{\frac{R}{2(1-t)}}-1}{E} \triangleq C_{4}\right), Y \geq \max \left(C_{3}, C_{4}\right), X+$ $\left.Y \geq C_{0}\right\}$.

1) For $t \leq \frac{1}{2}$ : we have $C_{3} \geq C_{4}$, and channel gain pair $(X, Y)$ is in the set of $\left\{(X, Y) \mid X \geq C_{3}, Y \geq C_{3}, X+Y \geq\right.$ $\left.C_{0}\right\}$. This is similar to Fig. 2, except that $C_{1}$ and $C_{0}^{*}$ are replaced by $C_{3}$ and $C_{0}$, respectively. Since $2 C_{3} \leq C_{0}$, i.e., $2 \cdot\left(2^{\frac{R}{2 t}}-1\right) \leq 2^{\frac{R}{t}}-1$ always holds if $0 \leq t \leq \frac{1}{2}$, only the event shown in Fig. 2(b) will occur. Thus, the outage probability of the system is given by:

$$
\begin{aligned}
P_{\text {out }(\alpha=1 / 2, \mathrm{t})} & =1-\frac{\Omega_{h}}{\Omega_{h}-\Omega_{f}} e^{-\frac{1}{\Omega_{h}} C_{0}+\left(-\frac{1}{\Omega_{f}}+\frac{1}{\Omega_{h}}\right) C_{3}} \\
& +\frac{\Omega_{f}}{\Omega_{h}-\Omega_{f}} e^{-\frac{1}{\Omega_{f}} C_{0}+\left(-\frac{1}{\Omega_{h}}+\frac{1}{\Omega_{f}}\right) C_{3}} .
\end{aligned}
$$

If $\Omega_{h}=\Omega_{f}$, the outage probability of the system is:

$$
P_{\text {out }(\alpha=1 / 2, \mathrm{t})}=1-e^{-\frac{1}{\Omega_{h}} C_{0}}-\frac{1}{\Omega_{h}}\left(C_{0}-2 C_{3}\right) e^{-\frac{1}{\Omega_{h}} C_{0}} .
$$

2) For $t>\frac{1}{2}$ : we have $C_{3}<C_{4}$. Hence, channel gain pair $(X, Y)$ satisfies $\left\{(X, Y) \mid X \geq C_{4}, Y \geq C_{4}, X+Y \geq C_{0}\right\}$, as in Fig. 2, with $C_{1}$ and $C_{0}^{*}$ replaced by $C_{4}$ and $C_{0}$, respectively. In the same way, channel gain pair $(X, Y)$ is in Fig. 2(a) when $2 C_{4}>C_{0}$, i.e., $2 \cdot\left(2^{\frac{R}{2(1-t)}}-1\right)>2^{\frac{R}{t}}-1$, otherwise, in Fig. 2(b). This inequality is transcendental which does not have exact analytical solution. However, note that the function $2^{\frac{R}{t}}-2 \cdot 2^{\frac{R}{2(1-t)}}+1$ at a given $R$ is monotonically decreasing with respect to $t$. So there exists a unique zero and we denote it as $t_{0}$. Hence, the outage probability of the system is:

$$
= \begin{cases}P_{\operatorname{out}(\alpha=1 / 2, \mathrm{t})} & t_{0} \leq t \leq 1 \\ \left.1-\frac{\Omega_{h}}{\Omega_{h}-\Omega_{f}} e^{-\frac{1}{\Omega_{h}} C_{0}+\left(-\frac{1}{\Omega_{f}}\right) C_{4}}, \frac{1}{\Omega_{h}}\right) C_{4} & \\ +\frac{\Omega_{f}}{\Omega_{h}-\Omega_{f}} e^{-\frac{1}{\Omega_{f}} C_{0}+\left(-\frac{1}{\Omega_{h}}+\frac{1}{\Omega_{f}}\right) C_{4}}, & \frac{1}{2}<t \leq t_{0}\end{cases}
$$

when $\Omega_{h}=\Omega_{f}$, the outage probability of the system is as follows:

$$
P_{\text {out }(\alpha=1 / 2, t)}= \begin{cases}1-e^{-\frac{2}{\Omega_{h}} C_{4}}, & t_{0} \leq t \leq 1 \\ 1-e^{-\frac{1}{\Omega_{h}} C_{0}} & \\ -\frac{1}{\Omega_{h}}\left(C_{0}-2 C_{4}\right) e^{-\frac{1}{\Omega_{h}} C_{0}}, & \frac{1}{2} \leq t \leq t_{0}\end{cases}
$$

\section{Arbitrary time sharing and rate allocation}

Finally, let time-sharing parameter and rate allocation parameter take any values. Using the similar approach as in the previous two subsections, we can obtain a generic outage probability formula as:

$$
\begin{aligned}
& P_{\text {out }(\alpha, \mathrm{t})} \\
& \qquad \begin{aligned}
& 1-e^{-\frac{1}{\Omega_{h}} C_{X}-\frac{1}{\Omega_{f}} C_{Y}}, \\
& \text { if } \max \left(\frac{\alpha}{\mathrm{t}}, \frac{1-\alpha}{1-\mathrm{t}}\right)+\max \left(\frac{\alpha}{1-\mathrm{t}}, \frac{1-\alpha}{\mathrm{t}}\right)>\frac{1}{\mathrm{t}} \\
& 1-\frac{\Omega_{h}}{\Omega_{h}-\Omega_{f}} e^{-\frac{1}{\Omega_{h}} C_{0}+\left(-\frac{1}{\Omega_{f}}+\frac{1}{\Omega_{h}}\right) C_{Y}} \\
&+\frac{\Omega_{f}}{\Omega_{h}-\Omega_{f}} e^{-\frac{1}{\Omega_{f}} C_{0}+\left(-\frac{1}{\Omega_{h}}+\frac{1}{\Omega_{f}}\right) C_{X}}, \\
& \text { if } \max \left(\frac{\alpha}{\mathrm{t}}, \frac{1-\alpha}{1-\mathrm{t}}\right)+\max \left(\frac{\alpha}{1-\mathrm{t}} \cdot \frac{1-\alpha}{\mathrm{t}}\right) \leq \frac{1}{\mathrm{t}}
\end{aligned}
\end{aligned}
$$

In the case of $\Omega_{h}=\Omega_{f}$ :

$$
\begin{aligned}
& P_{\text {out }(\alpha, \mathrm{t})} \\
& =\left\{\begin{aligned}
& 1-e^{-\frac{1}{\Omega_{h}}\left(C_{X}+C_{Y}\right)}, \text { if } \max \left(\frac{\alpha}{t}, \frac{1-\alpha}{1-t}\right)+\max \left(\frac{\alpha}{1-t}, \frac{1-\alpha}{t}\right)>\frac{1}{t} \\
& 1-e^{-\frac{1}{\Omega_{h}} C_{0}}-\frac{1}{\Omega_{h}}\left(C_{0}-C_{X}\right)-C_{Y} e^{-\frac{1}{\Omega_{h}} C_{0}}, \\
& \text { if } \max \left(\frac{\alpha}{t}, \frac{1-\alpha}{1-t}\right)+\max \left(\frac{\alpha}{1-t}, \frac{1-\alpha}{t}\right) \leq \frac{1}{t}
\end{aligned}\right.
\end{aligned}
$$

where $C_{X}=\max \left(C_{X 1}, C_{X 2}\right), C_{Y}=\max \left(C_{Y 1}, C_{Y 2}\right)$.

Before leaving this section, we briefly discuss the conditions to reach the minimal outage probability. By observing the events (9), the constraint on $R$ can be rewritten as:

$$
\begin{aligned}
& R \leq \min ( \frac{t}{\alpha} \log _{2}(1+E X), \frac{1-t}{1-\alpha} \log _{2}(1+E X), \\
& \frac{t}{1-\alpha} \log _{2}(1+E Y), \frac{1-t}{\alpha} \log _{2}(1+E Y), \\
&\left.t \log _{2}(1+E X+E Y)\right) .
\end{aligned}
$$

As a result, under equal time-sharing condition, choosing $\alpha=$ $1 / 2$ can minimize outage probability regardless of the values of $\Omega_{h}$ and $\Omega_{f}$. Similarly, letting $t=1 / 2$ can achieve the minimal outage probability when the rate allocation is fair, for any $\Omega_{h}$ and $\Omega_{f}$.

\section{FINITE-SNR DMT}

At finite SNR, we recall the definitions of multiplexing gain $r$ and diversity gain $d$ in [11] as:

$$
\begin{gathered}
r=\frac{R}{\log _{2}(1+g \gamma)}, \\
d(r, \gamma)=-\frac{\partial \ln P_{\text {out }}(r, \gamma)}{\partial \ln \gamma} .
\end{gathered}
$$

The multiplexing gain $r$ is the ratio of rate $R$ to the capacity of an AWGN channel at SNR $\gamma$ with array gain $g$ chosen as the number of receive antennas at low to medium SNR. Furthermore, for a given $r$, diversity gain is defined as the negative slope of the log-log plot of outage probability versus SNR. In this paper, since the noise is assumed to have unit 
variance, $d$ becomes the function of both multiplexing gain $r$ and transmission power $E$ as follows:

$$
d(r, E)=-\frac{\partial \ln P_{\text {out }}(r, E)}{\partial \ln E}=-\frac{E}{P_{\text {out }}(r, E)} \frac{\partial P_{\text {out }}(r, E)}{\partial E}
$$

As in Section III, we derive finite-SNR DMT for different values of time sharing and rate allocation in the following three subsections.

\section{A. Equal time sharing and arbitrary rate allocation}

Substituting (21) into $C_{1}$ and $C_{0}^{*}$ defined in Section IIIA for equal time sharing, we have $C_{1}=\frac{(1+E)^{2 \alpha r}-1}{E}$ and $C_{0}^{*}=\frac{(1+E)^{2 r}-1}{E}$. Then, substituting (12) and (13) into (23), and taking derivative, we can obtain:

$$
d(r, E)_{(\alpha, t=1 / 2)}= \begin{cases}\frac{\left(\frac{1}{\Omega_{h}}+\frac{1}{\Omega_{f}}\right) C_{1 \_} d e r}{1-e^{\left(\frac{1}{\Omega_{x}}+\frac{1}{\Omega_{f}}\right) C_{1}}}, & \alpha_{0} \leq \alpha \leq 1 \\ \frac{d_{1}-d_{2}}{P_{\text {out }(\alpha, \mathrm{t}=1 / 2)}}, & \frac{1}{2} \leq \alpha \leq \alpha_{0}\end{cases}
$$

for $\Omega_{h} \neq \Omega_{f}$, and

$$
\begin{aligned}
& d(r, E)_{(\alpha, t=1 / 2)} \\
& = \begin{cases}\frac{\frac{2}{\Omega_{h}} C_{1 \_} d e r}{1-e^{\frac{2}{\Omega_{h}} C_{1}},} & \alpha_{0} \leq \alpha \leq 1 \\
\frac{\frac{1}{\Omega_{h}}\left(2 C_{1 \_} d e r+\frac{1}{\Omega_{h}}\left(C_{0}^{*}-2 C_{1}\right) C_{0}^{*} d e r\right)}{1-e^{\frac{1}{\Omega_{h}} C_{0}^{*}}+\frac{1}{\Omega_{h}}\left(C_{0}^{*}-2 C_{1}\right)}, & \frac{1}{2} \leq \alpha \leq \alpha_{0}\end{cases}
\end{aligned}
$$

for $\Omega_{h}=\Omega_{f}$. Here,

$$
\begin{aligned}
d_{1}= & \frac{\Omega_{h}}{\Omega_{h}-\Omega_{f}} e^{-\frac{1}{\Omega_{h}} C_{0}^{*}+\left(-\frac{1}{\Omega_{f}}+\frac{1}{\Omega_{h}}\right) C_{1}} \\
& \left(-\frac{1}{\Omega_{h}} C_{0}^{*} d e r+\left(-\frac{1}{\Omega_{f}}+\frac{1}{\Omega_{h}}\right) C_{1} d e r\right), \\
d_{2}= & \frac{\Omega_{f}}{\Omega_{h}-\Omega_{f}} e^{-\frac{1}{\Omega_{f}} C_{0}^{*}+\left(-\frac{1}{\Omega_{h}}+\frac{1}{\Omega_{f}}\right) C_{1}} \\
& \left(-\frac{1}{\Omega_{f}} C_{0}^{*} d e r+\left(-\frac{1}{\Omega_{h}}+\frac{1}{\Omega_{f}}\right) C_{1 \_} d e r\right) .
\end{aligned}
$$

$C_{1 \_} d e r$ and $C_{0}^{*}$ der are the products of the derivatives of $C_{1}$ and $C_{0}^{*}$ multiplied by $E$ :

$$
\begin{aligned}
& C_{1} \_d e r=\frac{2 \alpha r(1+E)^{2 \alpha r-1} E-(1+E)^{2 \alpha r}+1}{E}, \\
& C_{0 \_}^{*} d e r=\frac{2 r(1+E)^{2 r-1} E-(1+E)^{2 r}+1}{E} .
\end{aligned}
$$

When $E$ tends to infinity, it can be shown that (24) and (25) approach:

$\underset{E \rightarrow \infty}{d(r, E)_{(\alpha, t=1 / 2)}}= \begin{cases}1-2 \alpha r, & 0 \leq r \leq \frac{1}{2 \alpha}, \text { if } \alpha_{0}<\alpha \leq 1 \\ 1-2 r, & 0 \leq r \leq \frac{1}{2}, \text { if } \frac{1}{2} \leq \alpha \leq \alpha_{0}\end{cases}$

It is seen that the maximal multiplexing gain is smaller than one due to the additional sum-rate constraint to enforce relay node to successfully decode both signals from two sources in MA phase.

From (24) and (25), we let $r \rightarrow 0$. Then, we can obtain the maximal diversity gain as:

$$
\underset{r \rightarrow 0}{d(r, E)}=1-\frac{E}{(1+E) \ln (1+E)} .
$$

The asymptotic result in (27) agrees with the conventional point-to-point single-antenna link in [11].

\section{B. Arbitrary time sharing and equal rate allocation}

Using the same method as in the previous subsection, we can get finite-SNR DMT under fair rate allocation:

$$
d(r, E)_{(\alpha=1 / 2, t)}= \begin{cases}\frac{d_{3}-d_{4}}{P_{\text {out }(\alpha=1 / 2, \mathrm{t})}}, & 0 \leq t \leq \frac{1}{2} \\ \frac{d_{5}-d_{6}}{P_{\text {out }(\alpha=1 / 2, \mathrm{t})}}, & \frac{1}{2}<t \leq t_{0} \\ \frac{\left(\frac{1}{\Omega_{h}}+\frac{1}{\Omega_{f}}\right) C_{4} d e r}{1-e^{\left(\frac{1}{\Omega_{h}}+\frac{1}{\Omega_{f}}\right) C_{4}}}, & t_{0}<t \leq 1\end{cases}
$$

for $\Omega_{h} \neq \Omega_{f}$, and

$$
\begin{aligned}
& d(r, E)_{(\alpha=1 / 2, t)} \\
& = \begin{cases}\frac{\frac{1}{\Omega_{h}}\left(2 C_{3 \_} d e r+\frac{1}{\Omega_{h}}\left(C_{0}-2 C_{3}\right) C_{0 \_} d e r\right)}{1-e^{\frac{1}{\Omega_{h}} C_{0}}+\frac{1}{\Omega_{h}}\left(C_{0}-2 C_{3}\right)}, & 0 \leq t \leq \frac{1}{2} \\
\frac{\frac{1}{\Omega_{h}}\left(2 C_{4 \_} d e r+\frac{1}{\Omega_{h}}\left(C_{0}-2 C_{4}\right) C_{0 \_} d e r\right)}{1-e^{\frac{1}{\Omega_{h}} C_{0}}+\frac{1}{\Omega_{h}}\left(C_{0}-2 C_{4}\right)}, & \frac{1}{2}<t \leq t_{0} \\
\frac{\frac{2}{\Omega_{h}} C_{4 \_} d e r}{1-e^{\frac{2}{\Omega_{h}} C_{4}}}, & t_{0}<t \leq 1\end{cases}
\end{aligned}
$$

for $\Omega_{h}=\Omega_{f}$. Here coefficients $d_{3}$ and $d_{5}$ have the same form as $d_{1}$ except that $C_{0}^{*}$ is replaced by $C_{0}, C_{1}$ is replaced by $C_{3}$ and $C_{4}$, respectively, and $C_{1 \_} d e r$ is replaced by $C_{3 \_} d e r$ and $C_{4 \_}$der, correspondingly. Similarly, $d_{4}$ and $d_{6}$ have the same form as $d_{2}$ with $C_{0}^{*}$ replaced by $C_{0}, C_{1}$ replaced by $C_{3}$ and $C_{4}$, respectively, and $C_{1 \_} d e r$ replaced by $C_{3 \_} d e r$ and $C_{4} \_d e r$, correspondingly. Furthermore, $C_{3 \_} d e r$ and $C_{4 \_} d e r$ are the products of derivatives of $C_{3}$ and $C_{4}$ multiplied by $E$, respectively.

When $E$ tends to infinity, we can get the DMT at asymptotically high SNR as follows:

$$
\begin{aligned}
& d(r, E)_{(\alpha=1 / 2, t)} \\
& = \begin{cases}1-\frac{r}{t}, & 0 \leq r \leq t \\
1-\frac{r}{2(1-t)}, & 0 \leq r \leq 2(1-t) \\
& \text { if } 0 \leq \mathrm{t} \leq \frac{1}{2} \text { or } \max \left(\frac{1}{2}, \mathrm{t}_{0}\right)<\mathrm{t} \leq \frac{2}{3}\end{cases}
\end{aligned}
$$

\section{Arbitrary time sharing and rate allocation}

In the most general case where the time sharing and rate allocation are arbitrary, the finite-SNR DMT formula can be 
shown as

$$
\begin{aligned}
& d(r, E)_{(\alpha, t)} \\
& = \begin{cases}\frac{\frac{1}{\Omega_{h}} C_{X_{-}} d e r+\frac{1}{\Omega_{f}} C_{Y_{-}} \text {der }}{1-e^{\frac{1}{\Omega_{h}} C_{X}+\frac{1}{\Omega_{f}} C_{Y}},} \\
\text { if } \max \left(\frac{\alpha}{t}, \frac{1-\alpha}{1-t}\right)+\max \left(\frac{\alpha}{1-t}, \frac{1-\alpha}{t}\right)>\frac{1}{t} \\
\frac{d_{7}-d_{8}}{P_{\text {out }(\alpha, \mathrm{t})}}, & \text { if } \max \left(\frac{\alpha}{t}, \frac{1-\alpha}{1-t}\right)+\max \left(\frac{\alpha}{1-t}, \frac{1-\alpha}{t}\right) \leq \frac{1}{t}\end{cases}
\end{aligned}
$$

for $\Omega_{h} \neq \Omega_{f}$, and

$$
\begin{aligned}
& d(r, E)_{(\alpha, t)} \\
& =\left\{\begin{array}{c}
\frac{\frac{1}{\Omega_{h}}\left(C_{X_{-}} \text {der }+C_{Y_{-}} \text {der }\right)}{1-e^{\frac{1}{\Omega_{h}}\left(C_{X}+C_{Y}\right)}} \\
\text { if } \max \left(\frac{\alpha}{t}, \frac{1-\alpha}{1-t}\right)+\max \left(\frac{\alpha}{1-t}, \frac{1-\alpha}{t}\right)>\frac{1}{t} \\
\frac{1}{\frac{1}{\Omega_{h}}\left(C_{X_{-}} \text {der }+C_{Y_{-}} d e r\right)+\frac{1}{\Omega_{h}}\left(C_{0}-C_{X}-C_{Y}\right) C_{0 \_} d e r} \\
1-e^{\frac{1}{\Omega_{h}} C_{0}}+\frac{1}{\Omega_{h}}\left(C_{0}-C_{X}-C_{Y}\right) \\
\text { if } \max \left(\frac{\alpha}{t}, \frac{1-\alpha}{1-t}\right)+\max \left(\frac{\alpha}{1-t}, \frac{1-\alpha}{t}\right) \leq \frac{1}{t}
\end{array}\right.
\end{aligned}
$$

for $\Omega_{h}=\Omega_{f}$, where $C_{Y \_}$der and $C_{X \_}$der are the products of derivatives of $C_{Y}$ and $C_{X}$ multiplied by $E$, and

$$
\begin{aligned}
d_{7}= & \frac{\Omega_{h}}{\Omega_{h}-\Omega_{f}} e^{-\frac{1}{\Omega_{h}} C_{0}+\left(-\frac{1}{\Omega_{f}} C_{X}+\frac{1}{\Omega_{h}} C_{Y}\right)} \\
& \left(-\frac{1}{\Omega_{h}} C_{0 \_} d e r-\frac{1}{\Omega_{f}} C_{X \_} d e r+\frac{1}{\Omega_{h}} C_{Y \_} d e r\right), \\
d_{8}= & \frac{\Omega_{f}}{\Omega_{h}-\Omega_{f}} e^{-\frac{1}{\Omega_{f}} C_{0}+\left(-\frac{1}{\Omega_{h}} C_{X}+\frac{1}{\Omega_{f}} C_{Y}\right)} \\
& \left(-\frac{1}{\Omega_{f}} C_{0 \_} d e r-\frac{1}{\Omega_{h}} C_{X \_} d e r+\frac{1}{\Omega_{f}} C_{Y \_} d e r\right) .
\end{aligned}
$$

\section{NUMERICAL RESULTS}

This section presents some numerical results of outage probability and finite-SNR DMT. We assume that all the three terminals are in a straight line and the relay is between $S_{1}$ and $S_{2}$. The distance between $S_{1}$ and $S_{2}$ is fixed at 1. Let $D$ and $1-D$ represent the distances from $\mathrm{S}_{1}$ to $\mathrm{R}$ and from $\mathrm{S}_{2}$ to $\mathrm{R}$, respectively. The path loss factor as in urban areas is assumed. Thus, $\Omega_{h}$ and $\Omega_{f}$ can be expressed as a function of $D$ : $\Omega_{h}=D^{-4}$ and $\Omega_{f}=(1-D)^{-4}$. For comparison, the outage probability and finite-SNR DMT results for twophase AF protocol derived in [12] are also shown. Note that the results of AF protocol are for equal time sharing and equal rate allocation.

Fig. 3 compares the outage probabilities and finite-SNR DMTs of AF and DF under equal rate allocation and equal time sharing with $D_{1}=0.3$. It is seen that when the multiplexing gain $r$ is small $(r \leq 0.5)$, or $E$ is small enough, the outage probability of DF is better. However, when $r>0.5$ and $E$ is large enough, AF outperforms. In term of finiteSNR DMT, obviously, AF is always higher than DF regardless of values of SNR. For each protocol, its finite-SNR DMT converges to the asymptotically high $\mathrm{SNR}$ as $E$ increases.

Fig. 4 shows the results of varying rate allocation conditions, when $\alpha=0.5$, both outage probability and finite-SNR DMT achieve the optimum with equal time sharing. While Fig. 5
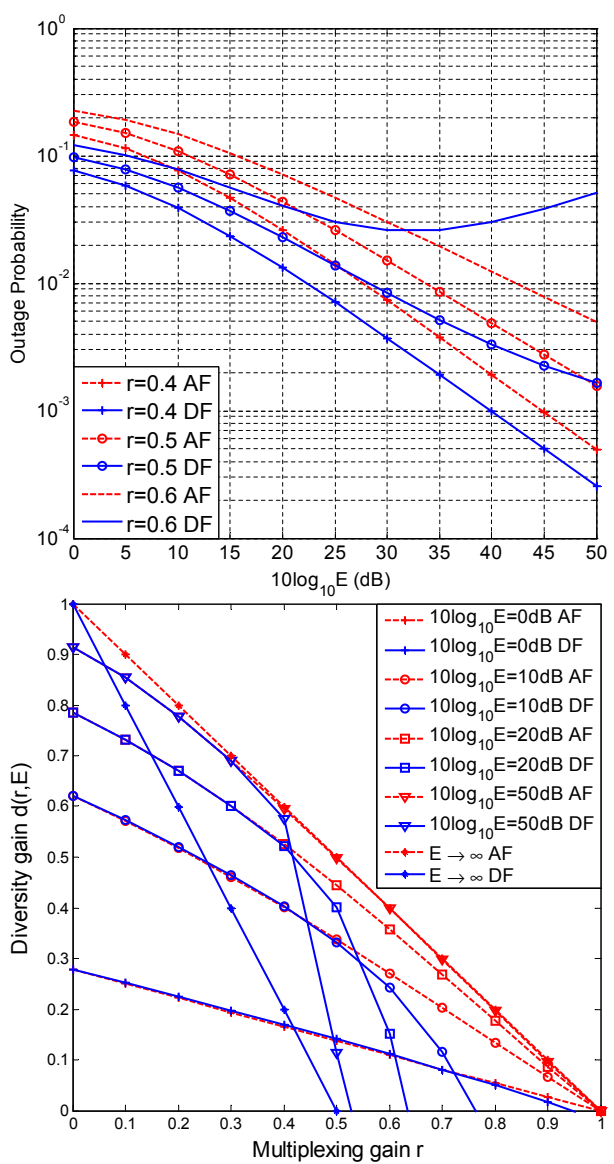

Fig. 3. Outage probabilities and finite-SNR DMTs of AF and DF under $t=0.5, \alpha=0.5, D=0.3$

presents the similar conclusion that $t=0.5$ makes both outage probability and finite-SNR DMT reach the optimal values under fair rate allocation condition.

Finally, Fig. 6 lists the optimal relay locations to minimize outage probability at different time sharing and rate allocation. It is seen that when $\alpha$ is around $1 / 2$ or $t$ is around $1 / 2$, the optimal relay location is always at $D=0.5$. In the special case of one-way relaying, i.e., $\alpha=0$ or 1 , the relay should be closer to the transmitting node when less time is spent on the first phase (hop) and vice versa.

\section{CONCLUSION}

This paper obtains closed-form expressions for outage probability and finite-SNR DMT with any rate allocation and time sharing in the two-phase DF TWRC. Through the analysis, we can conclude that in single-antenna TWRC, DF outperforms AF when either multiplexing gain or SNR is small enough, otherwise, AF is better in term of outage probability. However, finite-SNR DMT of DF is lower than that of AF regardless of SNR due to the sum-rate constraint for relay to fully decode. Moreover, numerical results demonstrate the optimum relay location under given conditions on rate allocation and time sharing. In the future work, the performance limits of multipleantenna TWRC with fading will be investigated. 


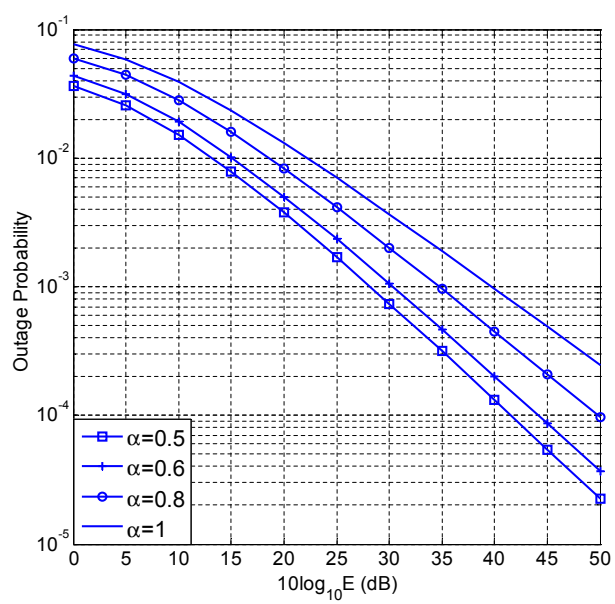

(a) $D=0.3, r=0.2$

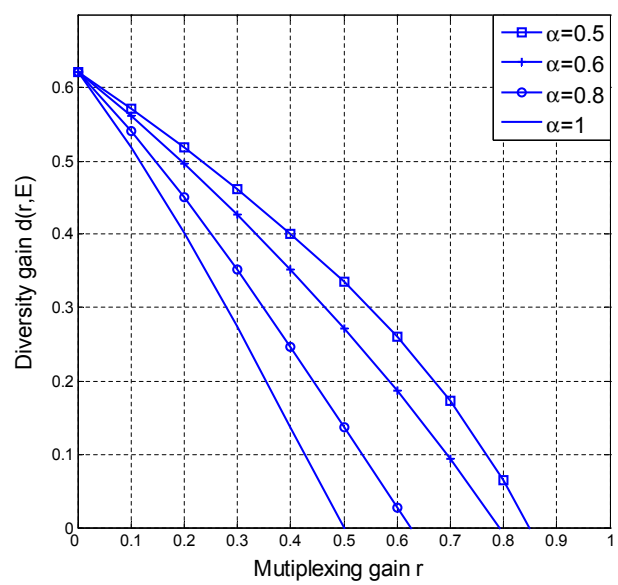

(b) $D=0.3,10 \log _{10} E=10 \mathrm{~dB}$

Fig. 4. Outage probability and finite-SNR DMT under equal time sharing and arbitrary rate allocation

\section{REFERENCES}

[1] P. Larsson, N. Johansson, and K. E. Sunell, "Coded bi-directional relaying", in Proc. IEEE VTC'06, May 2006.

[2] B. Rankov and A. Wittneben, "Spectral efficient protocols for half-duplex fading relay channels", IEEE Jour. Selec. Areas. in Comm., vol. 25, no. 2, pp. 379-389, Feb. 2007.

[3] T. J. Oechtering and H. Boche, "Optimal transmit strategies in multiantenna bidirectional relaying", in IEEE ICASSP, Honolulu, Hawaii, USA, vol.3, April 2007.

[4] P. Popovski and H. Yomo, "Bi-directional amplification of throughput in a wireless multi-hop network", in IEEE VTC, May 2006.

[5] T. J. Oechtering, C. Schnurr, I. Bjelakovic, and H. Boche, "Achievable rate region of a two phase biderectional relay channel", in Proc. Conf. Inf. Sci. and Sys. (CISS), Baltimore, MD, Mar. 2007.

[6] S. J. Kim, P. Mitran, and V. Tarokh, "Performance bounds for bidirectional coded cooperation protocols", IEEE Trans. Inf. Theory, vol. 54, no. 11, pp. 5235-5241, Nov. 2008.

[7] P. Popovski and H. Yomo, "Physical network coding in two-way wireless relay channels", in Proc. IEEE ICC, Glasgow, Scotland, June 2007.

[8] L. Zheng and D. N. C. Tse, "Diversity and multiplexing: a fundamental tradeoff in multiple-antenna channels", IEEE Trans. Inf. Theory, vol. 49, no. 5, pp. 1073-1096, May 2003.

[9] D. Gündüz, A. Goldsmith, and H. V. Poor, "MIMO two-way relay channel: diversity-multiplexing tradeoff analysis", in Asilomar Conf. Signals, Systems and Computers, Pacific Grove, CA, Oct. 2008.

[10] R. Vaze and R. W. Heath Jr., "On the capacity and diversity-multiplexing tradeoff of the two-way relay channel", IEEE Trans. Inf. Theory, submitted 2008

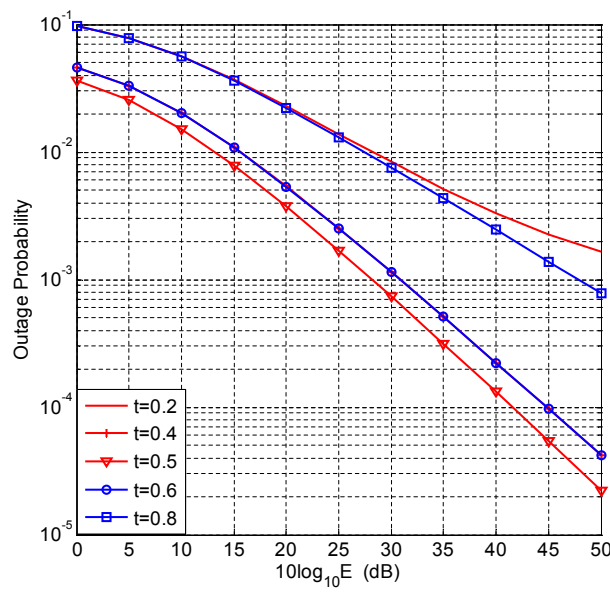

(a) $D=0.3, r=0.2$

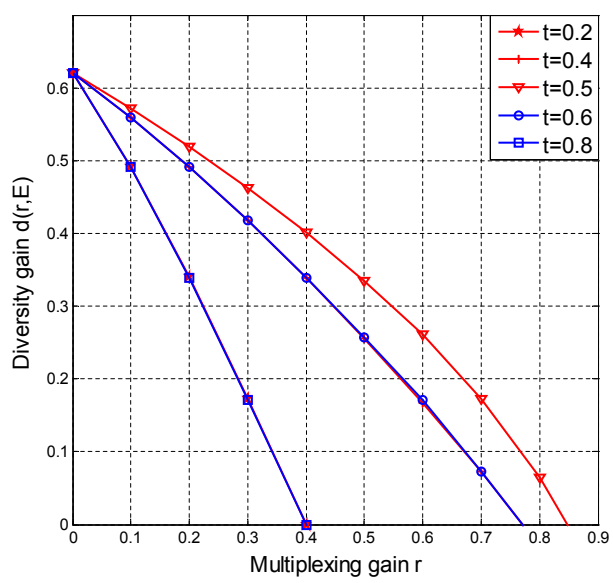

(b) $D=0.3,10 \log _{10} E=10 d B$

Fig. 5. Outage probability and finite-SNR DMT under arbitrary time sharing and fair rate allocation

\begin{tabular}{|c|c|c|c|c|c|c|c|c|c|}
\hline$\alpha_{\alpha} D^{t}$ & 0.1 & 0.2 & 0.3 & 0.4 & 0.5 & 0.6 & 0.7 & 0.8 & 0.9 \\
\hline 0 & 0.9 & 0.7 & 0.6 & 0.6 & 0.5 & 0.4 & 0.4 & 0.3 & 0.1 \\
\hline 0.1 & 0.8 & 0.7 & 0.6 & 0.6 & 0.5 & 0.4 & 0.4 & 0.3 & 0.2 \\
\hline 0.2 & 0.8 & 0.7 & 0.6 & 0.5 & 0.5 & 0.5 & 0.4 & 0.3 & 0.2 \\
\hline 0.3 & 0.7 & 0.6 & 0.6 & 0.5 & 0.5 & 0.5 & 0.4 & 0.4 & 0.3 \\
\hline 0.4 & 0.6 & 0.6 & 0.5 & 0.5 & 0.5 & 0.5 & 0.5 & 0.4 & 0.4 \\
\hline 0.5 & 0.5 & 0.5 & 0.5 & 0.5 & 0.5 & 0.5 & 0.5 & 0.5 & 0.5 \\
\hline 0.6 & 0.4 & 0.4 & 0.5 & 0.5 & 0.5 & 0.5 & 0.5 & 0.6 & 0.6 \\
\hline 0.7 & 0.3 & 0.4 & 0.4 & 0.5 & 0.5 & 0.5 & 0.6 & 0.6 & 0.7 \\
\hline 0.8 & 0.2 & 0.3 & 0.4 & 0.5 & 0.5 & 0.5 & 0.6 & 0.7 & 0.8 \\
\hline 0.9 & 0.2 & 0.3 & 0.4 & 0.4 & 0.5 & 0.6 & 0.6 & 0.7 & 0.8 \\
\hline 1 & 0.1 & 0.3 & 0.4 & 0.4 & 0.5 & 0.6 & 0.6 & 0.7 & 0.9 \\
\hline
\end{tabular}

Fig. 6. Optimal relay location to minimize outage probability under varying time sharing and rate allocation, $r=0.2,10 \log _{10} E=10 \mathrm{~dB}$

[11] R. Narasimhan, "Finite-SNR diversity-multiplexing tradeoff for correlated Rayleigh and Rician MIMO channels", IEEE Trans. Inf. Theory, vol. 52, no. 9, pp. 3965-3979, Sep. 2006.

[12] Z. H. Yi and Il-Min Kim, "Finite-SNR diversity-multiplexing tradeoff and optimum power allocation in bidirectional cooperative networks", IEEE Trans. Inf. Theory, submitted 2008. 